\title{
Größenentwicklung von Containerschiffen und Auswirkung auf die intermodale Transportkette
}

\author{
PROF. DR.-ING. GÜNTHER PAWELLEK \\ DIPL.-ING. AXEL SCHÖNKNECHT \\ TECHNISCHE UNIVERSITÄT HAMBURG-HARBURG, TECHNISCHE LOGISTIK
}

\begin{abstract}
Zusammenfassung
Gegenstand des vorliegenden Beitrages ist eine Methode zur Kosten- und Leistungsbewertung von Containerschiffen als Transportmittel des Hauptlaufes in intermodalen Transportketten für ISOContainer. Anlass bildet die permanente Größenentwicklung der Containerschiffe und die daraufhin ausgerichtete Infrastruktur- und Transportkettenentwicklung im Vor- und Nachlauf, die nicht risikofrei zu beurteilen ist. Mit der vorgestellten Methode wird deutlich, dass die Erfolgs- bzw. Misserfolgsfaktoren der Großcontainerschiffe fast nur noch in den Häfen und deren Hinterlandanbindungen zu suchen sind.
\end{abstract}

\begin{abstract}
Topic of this article is a method for cost- and performance benchmarking of containerships as means of transport of the main carriage in intermodal transportation chains for ISO-container. Occasion is the permanent growth of shipsize and upon that justified infrastructure and transport chain development in precarriage and onward carriage, which is not to be judged risk-free. The presented method will clarify that factors of success and failure depend nearly exclusive on the harbours and their hinterland connection.
\end{abstract}

\section{Einleitung}

Auf Grund des kontinuierlich steigenden Transportaufkommens im Seecontainerverkehr wird der Ruf nach größeren Containerschiffen von vielen Seiten immer lauter. Für etwa 21 Mrd. US \$ sind bereits Containerschiffe der neusten Generation bestellt [Dam06, S.60]. Mit immer größeren Schiffen könnten pro Containerschiffsumlauf deutlich mehr Container befördert werden als heute, die Umsätze werden steigen und damit die Gewinne, so die These [Ihl03]. Diese These wird sich jedoch nur bewahrheiten, wenn sich sowohl die Kosten unterproportional zur Schiffsgröße entwickeln, als auch die Anzahl der Containerschiffsumläufe nahezu gleich bleibt. Die Kombination der Kriterien aus Einnahmen, Kosten und Containerschiffsumläufen in einem bestimmten Zeitraum, kurz, die Tonnage- oder Slot-Produktivität, wurde bei der Diskussion der Schiffsgrößenentwicklung jedoch kaum geführt. Diese hängt von vielen logistischen Einflussfaktoren ab. Steigt die Slot-Produktivität mit zunehmender Schiffsgröße nicht, bzw. nimmt sie sogar ab, so erwirtschaften die GroßContainerschiffe letztendlich eine geringere Kapitalrendite. Tritt das ein, so ist eine ähnliche Entwicklung wie bei den Großtankern der 70er-Jahre zu befürchten [Stp02], die nahezu über Nacht verschrottet wurden. Bis dahin erstellte Infrastrukturen, zumeist finanziert durch die öffentliche Hand, erwiesen sich danach als sinnlos. Die bisherige Größenentwicklung der Ladekapazität der Containerschiffe in TEU ${ }^{1}$ zeigt Abbildung 1.

Um die Nachhaltigkeit des Containerschiffswachstums zu überprüfen, ist eine Methode notwendig, die das typische Betriebsverhalten der Containerschifffahrt abbildet. Mit der Methode soll aufgezeigt werden, von welchen wesentlichen Einflussfaktoren die Containerschiffsrentabilität und -produktivität abhängen und welche Rückwirkungen auf die Transportkette und ihre Schnittstellen bei steigender Schiffsgrößenentwicklung eintreten können bzw. welche Anpassungsmaßnahmen in den Häfen vorgenommen werden müssen, um die maximale Wirtschaftlichkeit von Großcontainerschiffen zu erreichen. Zunächst soll jedoch kurz der Zusammenhang zwischen Transportketten und dem Umlauf eines Containerschiffs erläutert werden.

\footnotetext{
${ }^{1}$ Twenty foot equivalent unit 


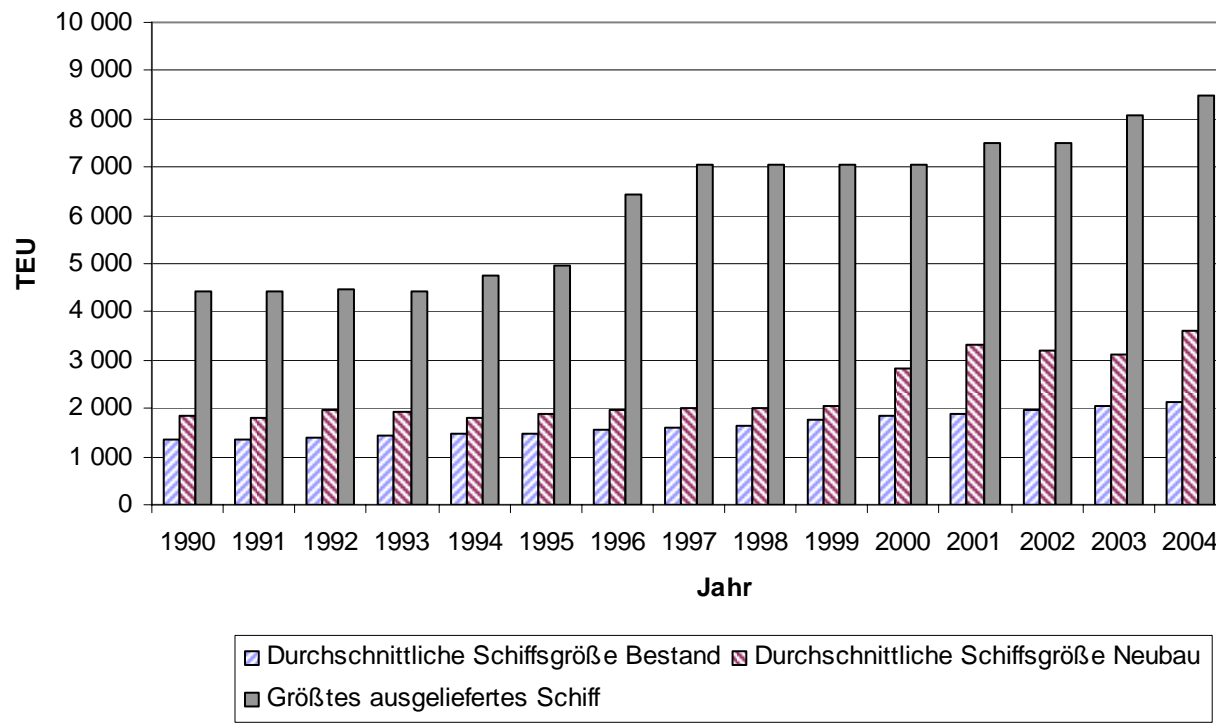

Abbildung 1:Entwicklung der durchschnittlichen und maximalen Containerschiffsgrößen [DAM-06-2, S.51 ]

\section{Abbildung der Transportkette und Einordnung des Containerschiffes}

Eine intermodale Transportkette ist als Transport von Gütern in ein und demselben Ladungsträger über mindestens zwei verschiedene Verkehrsträger definiert. Die hier betrachteten Transportketten sind in Vor-, Haupt- und Nachlauf separiert. Dadurch ist der Einsatz von mindestens drei Verkehrsträgern pro Transportkette erforderlich, wobei im Hauptlauf das Containerschiff zum Einsatz kommt. Zur Einordnung der Bedeutung der Containerschiffe in der Transportkette ist zunächst eine Darstellung erforderlich, die den Zusammenhang zwischen Transportkette als Folge von Transportprozessen für ein und dieselbe Ladungseinheit sowie den Einsatz eines Containerschiffes für viele verschiedene Ladungseinheiten im Rahmen vieler verschiedener Transportketten erläutert (Abb. 2).

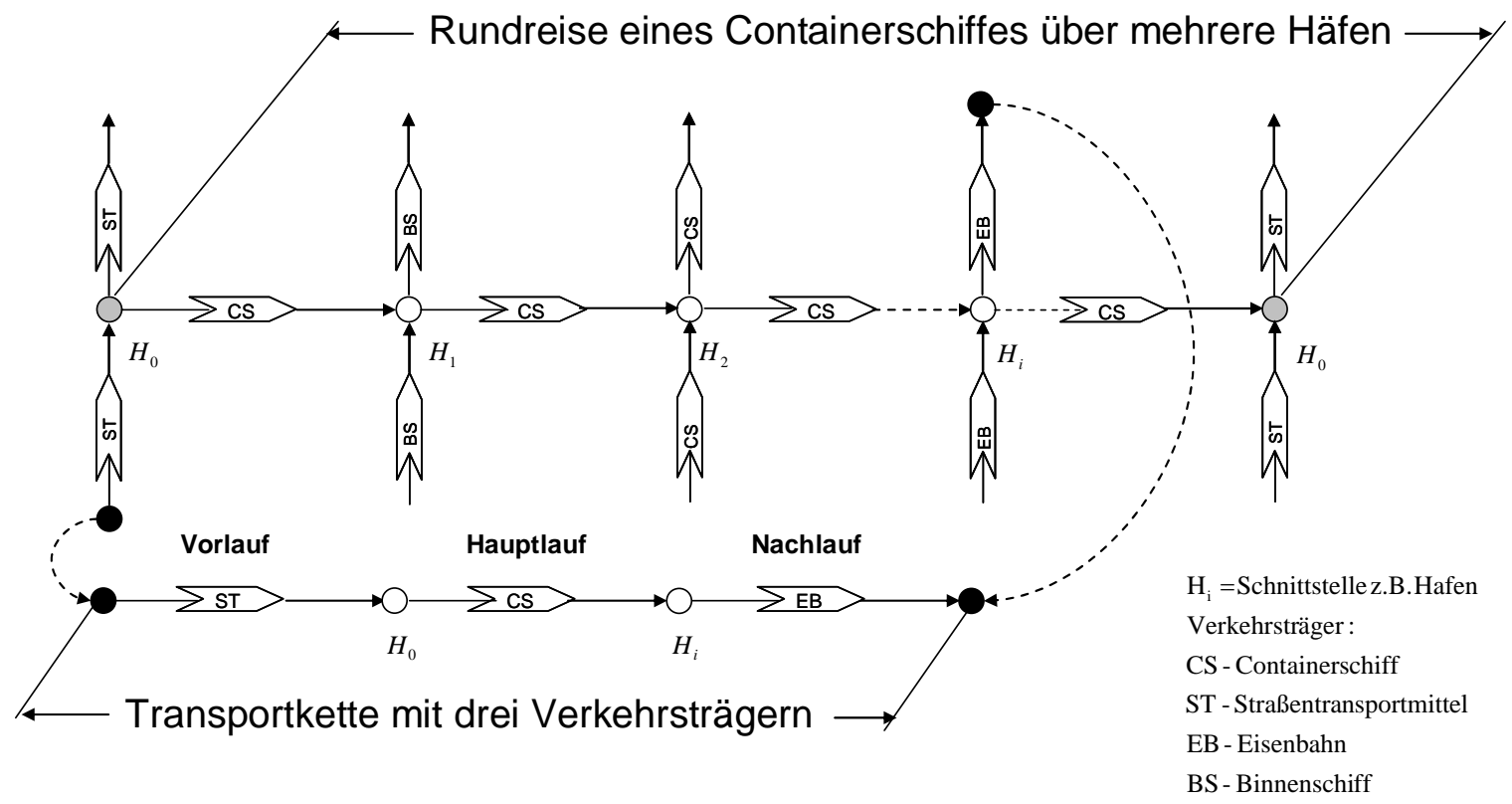

Abbildung 2: Zusammenhang zwischen Transportkette und Rundreise eines Containerschiffes 
Die operative Einsatzform des Containerschiffes in der Linienschifffahrt ist vornehmlich der Rundreisemodus. Rundreise ist hierbei ein Terminus der Containerlinienschifffahrt, der nichts mit der Rundreise aus dem Operations-Research zu tun hat. Innerhalb der hier betrachteten Rundreisen können dieselben Häfen auch mehrfach vorkommen. Start- und Endhafen sind aber identisch. Rundreisen der Linienschifffahrt finden in der Regel zwischen zwei Kontinenten statt. Asien-Nordamerika, Asien-Europa und Europa-Amerika sind dabei die wichtigsten Rundreiserelationen.

Jeder Hafen hat die Aufgabe die Ladungsgüter der verschiedenen Transportketten auf ein Containerschiff zu konzentrieren bzw. von diesem aus über die Transportketten zu verteilen. Der Einfluss der Containerschiffsgröße auf diese Aufgabe und die Anzahl der zu verbindenden Transportketten lässt sich mit Abbildung 3 beschreiben:

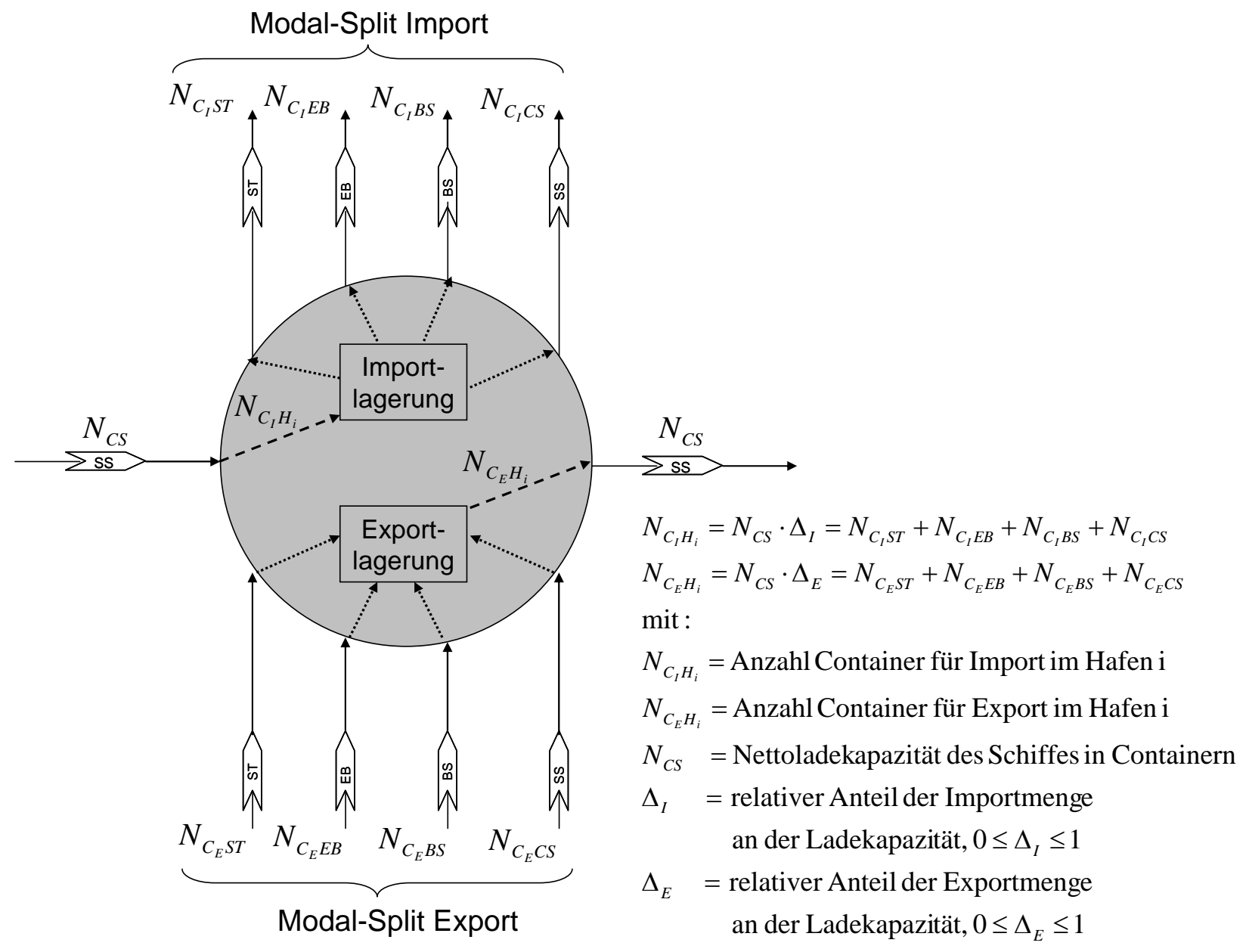

Abbildung 3: Einfluss der Containerschiffsgröße auf die Anzahl der zu verbindenden Transportketten in einem Hafen i

Demnach hängen die Anzahl der Transportketten und der zu handhabenden Container, die auf eine Schiffsabfahrt konzentriert oder von dieser aus verteilt werden müssen von der Nettoladekapazität $N_{C S}{ }^{2}$ des Schiffes sowie einem Faktor $\Delta$ ab. Der Faktor $\Delta$ gibt an, wie viel Container im Verhältnis zur Schiffladekapazität umgeschlagen werden. Er kann für den Im- und Exportbereich unterschiedlich ausfallen.

Die Nettoladekapazität $N_{C S}$ der Containerschiffe wächst permanent. Das ist bekannt. Es ist jedoch nicht bekannt, ob es sich hierbei um eine temporäre Erscheinung handelt, ähnlich wie bereits erwähnt die der Großtanker aus den 70er-Jahren oder ob dauerhaft mit sehr großen Containerschiffen in den Häfen zu rechnen ist. Als zu betrachtende Schiffe in dieser Untersuchung wird folgendes Schiffsgrößencluster gewählt (Tab. 1):

\footnotetext{
${ }^{2}$ die Nettoladekapazität eines Schiffes ist in der Regel kleiner als die reine Stellplatzkapazität eines Schiffes. Pro Stellplatz muss ein Gewicht von 14t eingerechnet werden [Cul98, S.189]. Damit wird die Tragfähigkeit eines Schiffes schon vor Ausnutzung aller Stellplätze erreicht.
} 
Tabelle 1: Definition des zu untersuchenden Schiffsgrößencluster

\begin{tabular}{|c|c|c|c|c|c|c|c|c|}
\hline Größencluster & $\begin{array}{c}\text { Tragfähigkeit } \\
\text { in dwt }\end{array}$ & $\begin{array}{c}\text { Stellplätze in } \\
\text { TEU }\end{array}$ & Nominal TEU & Referenz TEU & $\begin{array}{c}\text { Geschwindig- } \\
\text { keit in kn }\end{array}$ & $\begin{array}{c}\text { Antriebs- } \\
\text { leistung in } \\
\mathrm{kW}\end{array}$ & $\begin{array}{l}\text { Länge ü.a. } \\
\text { in } \mathrm{m}\end{array}$ & BRZ \\
\hline TEU 0-1.000 & 7.673 & 645 & 548 & 548 & 17 & 5.833 & 116 & 6.452 \\
\hline TEU 1.000-2.000 & 22.575 & 1.400 & 1.613 & 1.400 & 19 & 10.763 & 169 & 17.315 \\
\hline TEU 2.000-3.000 & 40.336 & 2.761 & 2.881 & 2.761 & 21 & 22.760 & 225 & 34.649 \\
\hline TEU 3.000-4.000 & 46.510 & 3.595 & 3.322 & 3.322 & 24 & 31.670 & 251 & 39.698 \\
\hline TEU 4.000-5.000 & 66.327 & 4.848 & 4.738 & 4.738 & 23 & 36.877 & 288 & 57.898 \\
\hline TEU 5.000-6.000 & 76.622 & 6.289 & 5.473 & 5.473 & 25 & 64.713 & 292 & 70.552 \\
\hline TEU 6.000-7.000 & 87.943 & 6.744 & 6.282 & 6.282 & 25 & 60.173 & 306 & 80.420 \\
\hline TEU 7.000-8.000 & 101.429 & 7.523 & 7.245 & 7.245 & 25 & 63.990 & 330 & 89.717 \\
\hline TEU 8.000-9.000 & 104.203 & 8.546 & 7.443 & 7.443 & 25 & 68.387 & 334 & 92.583 \\
\hline TEU $9.000-10.000$ & 108.956 & 9.216 & 7.783 & 7.783 & 25 & 70.346 & 346 & 103.498 \\
\hline TEU $10.000-11.000$ & 152.000 & 12.670 & 10.857 & 10.857 & 23 & 70.000 & 390 & 150.000 \\
\hline TEU 11.000-12.000 & 157.000 & 11.989 & 11.214 & 11.214 & 25 & 91.537 & 400 & 142.418 \\
\hline TEU 13.000 & 175.000 & 13.640 & 12.500 & 12.500 & 23 & 77.941 & 400 & 150.000 \\
\hline TEU 18.000 & 242.800 & 18.154 & 17.343 & 17.343 & 25 & 116.588 & 400 & 239.380 \\
\hline
\end{tabular}

Weiterhin besteht Forschungsbedarf darin, wie sich der Faktor $\Delta$ in Abhängigkeit der Containerschiffsgröße entwickelt und ob es dadurch Auswirkungen oder Anforderungen auf den Modal-Split der Verkehrsträger im Vor- und Nachlauf der Häfen gibt. Um diese Fragen zu beantworten wird die These aufgestellt, dass sich Containerschiffe über einen längeren Zeitraum in ihren Dimensionen so entwickeln, dass sie die maximale Wirtschaftlichkeit erzielen. Zur Abschätzung der Wirtschaftlichkeit unterschiedlicher Containerschiffsgrößen ist deshalb ein parametrierbares Kosten- und Einnahmemodell der Rundreise eines Containerschiffs notwendig, das im Folgenden vorgestellt wird.

\section{Kosten- und Einnahmenmodell eines Containerschiffes in der Linienschifffahrt}

Es wird vorangestellt, dass hier ein Modell gesucht wird, das die Wirtschaftlichkeit verschiedener Containerschiffsgrößen vergleicht und nicht die exakte Wirtschaftlichkeitsberechnung einer Rundreise widerspiegelt.

\subsection{Kostenmodell}

\subsubsection{Kostenmodell einer Rundreise}

Der Ansatz für das Kostenmodell wird zunächst über eine Rundreise getroffen die aus k Häfen besteht, wobei der Hafen $H_{0}$ und $H_{k}$ identisch sind und zwischen jedem Hafen eine Distanz $S_{i}$ zu überwinden ist (Abb. 4).

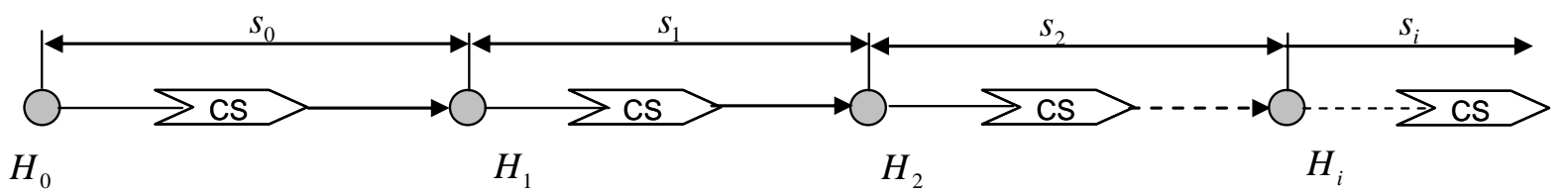

Abbildung 4: $\quad$ Abbildung einer Rundreise in der Containerlinienschifffahrt

Die Summe alle Teilseestrecken $S_{i}$ ist gleich die Rundreiselänge $S_{R}$. In jedem Hafen $H_{i}$ eine Menge Exportcontainer $N_{C_{E}}$ zu laden und eine Menge Importcontainer $N_{C_{I}}$ zu löschen, wobei sich die Container jeweils in 20' und 40' Container aufteilen können (Abb. 5).

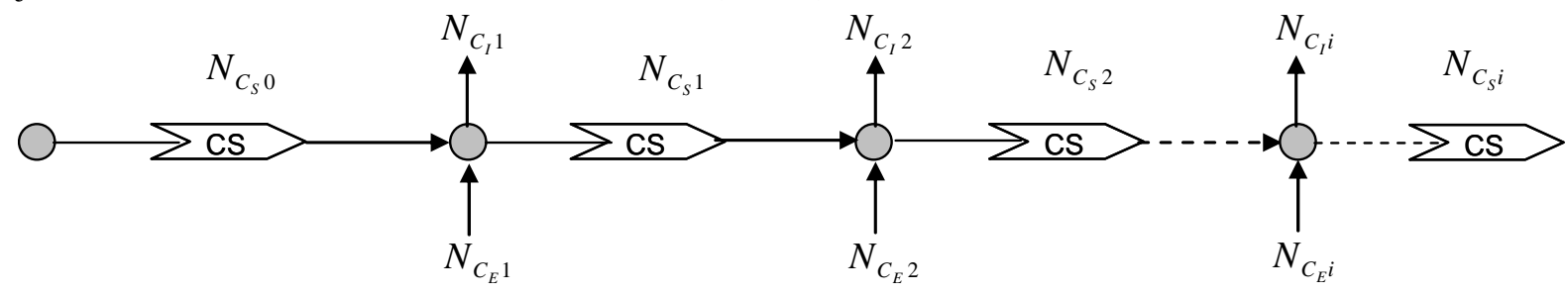

Abbildung 5: Containerumschlagaufkommen einer Rundreise 
Die Menge an beförderten Containern zwischen den Häfen $N_{C_{S} i}$ kann dabei nicht größer als die Ladekapazität des Schiffes $N_{C S}$ multipliziert mit einem Auslastungsfaktor $\alpha$ sein. Zusammenfassend gilt für das Containeraufkommen pro Hafen:

$$
\begin{aligned}
& N_{C_{S} i}=N_{C_{S} i-1}-N_{C_{I} i}+N_{C_{E} i} \\
& N_{C_{I} i}=N_{C_{I 20} i}+N_{C_{I 40} i} \\
& N_{C_{E} i}=N_{C_{E 20} i}+N_{C_{E 40} i} \\
& N_{C_{S} i} \leq N_{C S} \cdot \alpha \\
& 0 \leq \alpha \leq 1
\end{aligned}
$$

Die Nettoladekapazität $N_{C S}$ eines Schiffes in Containern hängt auch von der Verteilung zwischen 20' und 40' Containern ab. Diese Verteilung wird durch den TEU-Faktor $F_{\text {TEU }}$ ausgedrückt. Für eine Containermenge $N_{T E U}$ die nicht in Anzahl Containern sondern nur in TEU angegeben wird gilt.

$$
\begin{aligned}
& F_{T E U}=\frac{N_{T E U}}{N_{C}} ; 1 \leq F_{T E U} \leq 2 \\
& N_{T E U}=N_{C 20}+2 \cdot N_{C 40} \\
& F_{T E U}=\frac{N_{C 20}+2 \cdot N_{C 40}}{N_{C}}=1+\frac{N_{C 40}}{N_{C}} \\
& N_{C 40}=N_{C} \times\left(F_{T E U}-1\right) \\
& N_{C 20}=N_{C}-N_{40}
\end{aligned}
$$

Somit ist die Schiffsnettoladekapazität:

$$
N_{C S}=\frac{N T E U_{S}}{F_{T E U}}
$$

wobei $N_{T E U}$ nun die Schiffsladekapazität in Netto-TEU ist.

Mit diesen Randbedingungen lässt sich die Rundreisezeit $T_{R}$ eines Containerschiffes als Summe der reinen Seezeiten $T_{s i}$ und Hafenliegezeiten $T_{L i}$ berechnen (Abb. 6).

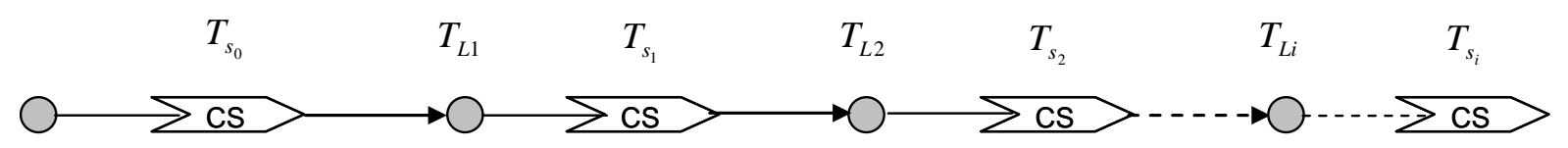

\section{Abbildung 6: Zeitkomponenten einer Rundreise}

Die Seezeit pro Teilstrecke $S_{i}$ wird hier vereinfachend wie folgt berechnet:

$$
T_{s_{i}}=\frac{s_{i}}{V_{s}}
$$

Die Hafenliegezeit setzt sich aus einer konstanten Anlauf- und Abfertigungszeit $T_{H i}$ sowie der containermengenabhängigen Umschlagzeit $T_{U i}$ zusammen. 
Bei der Berechnung der Umschlagzeit ist die spezifische Umschlagzeit $T_{U M}$ für einen Lade- oder Löschvorgang pro Containerbrücke sowie die Anzahl möglicher Containerbrücken $N_{C B}$, die an einem Containerschiff zum Einsatz kommen von Bedeutung. Diese können von Hafen zu Hafen schwanken. Der Einfluss schlechter Staupläne soll an dieser Stelle unberücksichtigt bleiben. Bisher kommen weltweit nur Einfachspiele in der Containerverladung vor.

Die Liegezeit eines Containerschiffes im Hafen i lässt sich somit ausdrücken durch:

$$
T_{L i}=T_{H i}+\left(N_{C_{I} i}+N_{C_{E} i}\right) \cdot \frac{T_{U_{M H i}}}{N_{C B_{H i}}}
$$

Mit den Zeit- und Mengenvorgaben einer Rundreise können nun die verschiedenen Kostenarten berechnet und zu Gesamtkosten $K_{R}$ aufaddiert werden. Im Rahmen dieser Betrachtung existieren fünf Kostenarten, die während einer Rundreise anfallen bzw. auf diese umgelegt werden müssen.

mit:

$$
K_{R}=K_{F R}+K_{V S R}+K_{V H R}+K_{U R}+K_{\text {sonst }}
$$

$K_{F R}=$ Fixkosten

$K_{V S R}=$ VariableKosten See

$K_{V H R}=$ VariableKosten Hafen

$K_{U R}=$ VariableKosten Umschlag

$K_{\text {sonst }}=$ sonstige Kosten z.B. Suezkanal

Die Fixkosten pro Rundreise setzen sich dabei aus einem spezifischen und schiffsgrößenabhängigen Kostenfaktor $K_{F S}\left[€ /\right.$ Zeit] und der Rundreisezeit $T_{R}$ zusammen:

$$
K_{F R}=K_{F S} \cdot T_{R}
$$

In analoger Weise werden die variablen Kosten See

$$
K_{V s_{i}}=K_{V S} \cdot T_{s_{i}} ; K_{V S R}=\sum_{1}^{k} K_{V s_{i}}
$$

und variablen Kosten Hafen

$$
K_{V H i}=K_{H S} \cdot T_{L i} ; K_{V H R}=\sum_{1}^{k} K_{V H i}
$$

berechnet. Die spezifischen Kostenfaktoren $K_{F S}, K_{V S}$ und $K_{H S}$ pro Schiffsgröße wurden in einer gesonderten Berechnungsmethode ermittelt. Bei den spezifischen Fixkosten $K_{F S}$ wurde dabei der Large-Scale oder Economy of Scale Effect nachgewiesen (Abb. 7). Bei allen anderen spezifischen Kostenfaktoren nicht. Weiterhin ist zu beachten, dass die Hafenkosten $K_{H S}$ in der Regel immer für angebrochene 24h berechnet werden. 


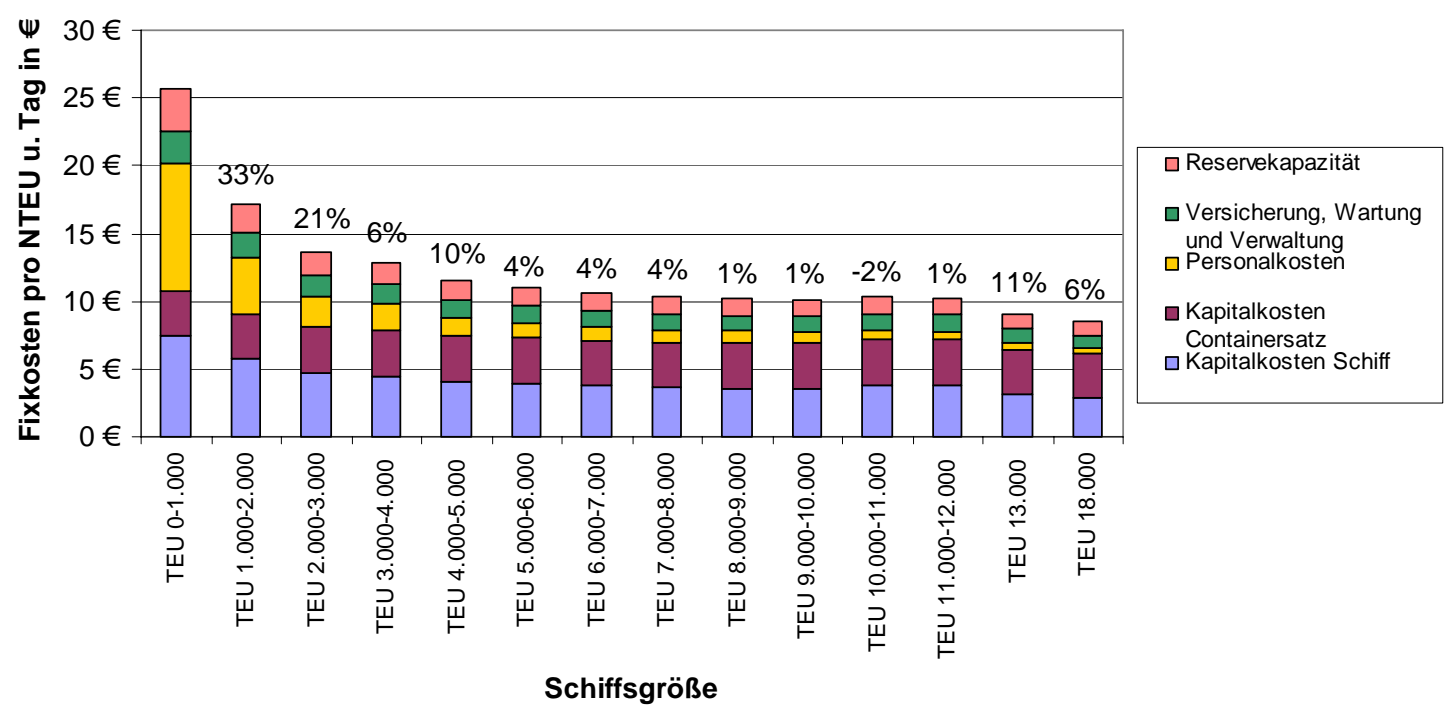

\section{Abbildung 7: Verlauf der Fixkosten $K_{F S} \quad$ in Abhängigkeit der Schiffsgröße mit relativer Änderung zum Vorgänger}

Bei den Umschlagkosten fallen für jeden umgeschlagenen Container in Abhängigkeit seiner Größe Kaitarife $K_{K A I_{20} H i}$ und $K_{K_{40} H i}$ an. Die Umschlagkosten pro Rundreise ergeben sich damit zu:

$$
\begin{aligned}
& K_{U i}=K_{U_{20} i}+K_{U_{40} i} \\
& K_{U_{20} i}=\left(N_{C_{I 20} i}+N_{C_{E 20} i}\right) \cdot K_{K A I_{20} H i} \\
& K_{U_{40} i}=\left(N_{C_{I 40} i}+N_{C_{E 40} i}\right) \cdot K_{K A I_{40} H i} \\
& K_{U R}=\sum_{1}^{k} K_{U i}
\end{aligned}
$$

Unter zu Grundlegung einer ganz konkreten Rundreise können nun die Kosten für verschiedene Schiffstypen pro Rundreise berechnet und verglichen werden. Beispielhaft ist das Ergebnis einer Europa-Asienreise in Abbildung 8 dargestellt.

Die bisherige Modellform bietet sich auch als Grundlage für eine Rundreisesimulation an, um insbesondere nur schwer geschlossen beschreibbare Effekte in den Häfen (z.B. unterschiedliche Umschlagverfahren) auf eine Rundreise einwirken zu lassen.

Die Parameter, z.B. Umschlagmengen, Kaitarife usw. müssen aber in diesem Modellansatz einem konkreten Hafen zugeordnet werden. Das ist jedoch nicht immer möglich bzw. bietet bei den Ergebnissen Anlass zur Diskussion, ob sich nicht unter bestimmten anderen Annahmen für einen Hafen die Ergebnisse ganz anders verhalten hätten. Da es aber unmöglich ist alle praktisch existierenden Rundreisen über alle Schiffsgrößen zu simulieren muss das Kostenmodell so weit abstrahiert werden, dass keine konkrete Rundreise zur Kostenberechnung als Grundlage notwendig ist. 


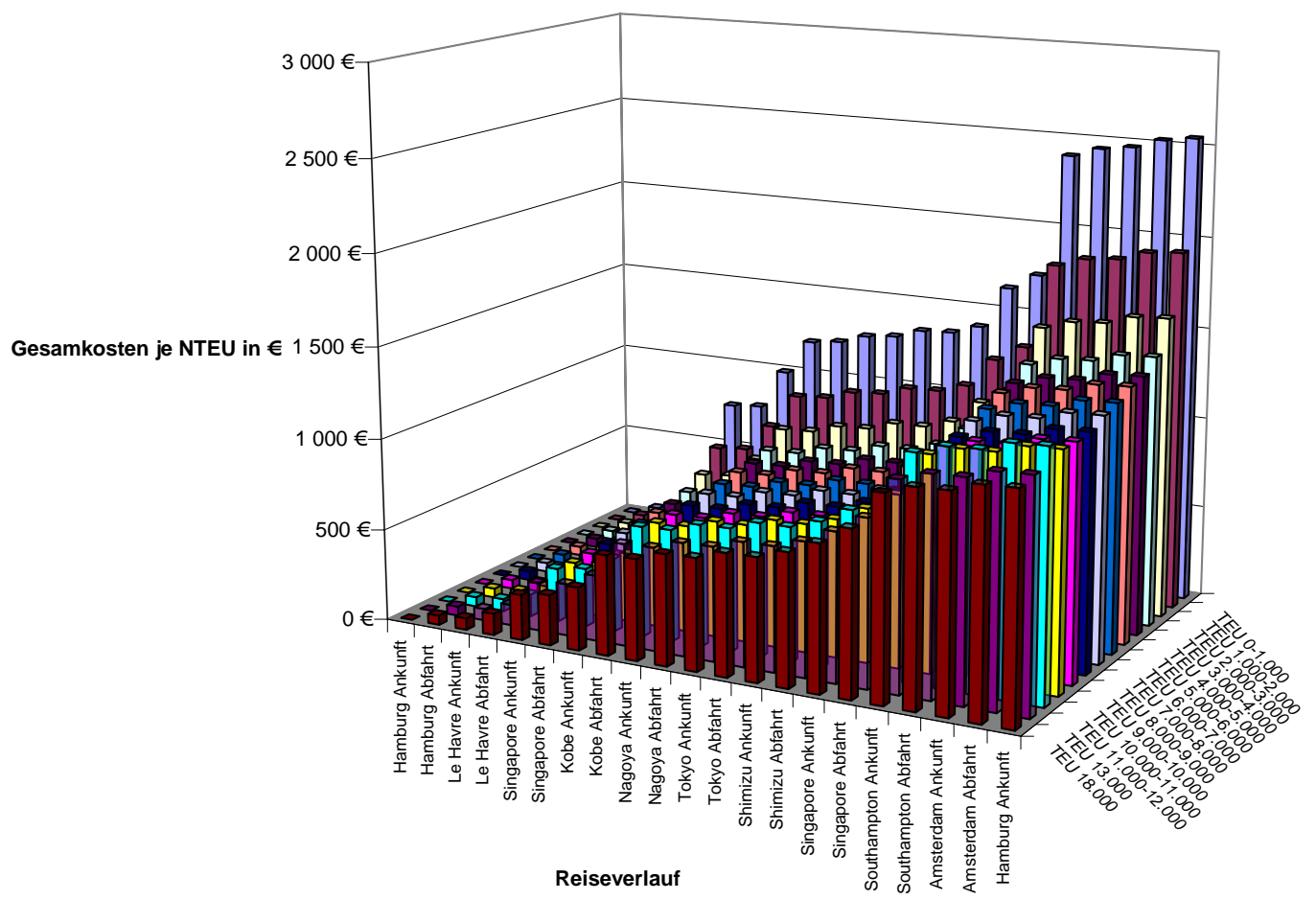

Abbildung 8: Beispiel für den Kostenverlauf einer Rundreise pro Stellplatz für die verschiedenen Schiffsgrößen des Schiffsgrößencluster

\subsubsection{Allgemeines Kostenmodell}

Die Menge aller umzuschlagenden Container einer Rundreise $N_{C_{R}}$ können wie folgt bestimmt werden:

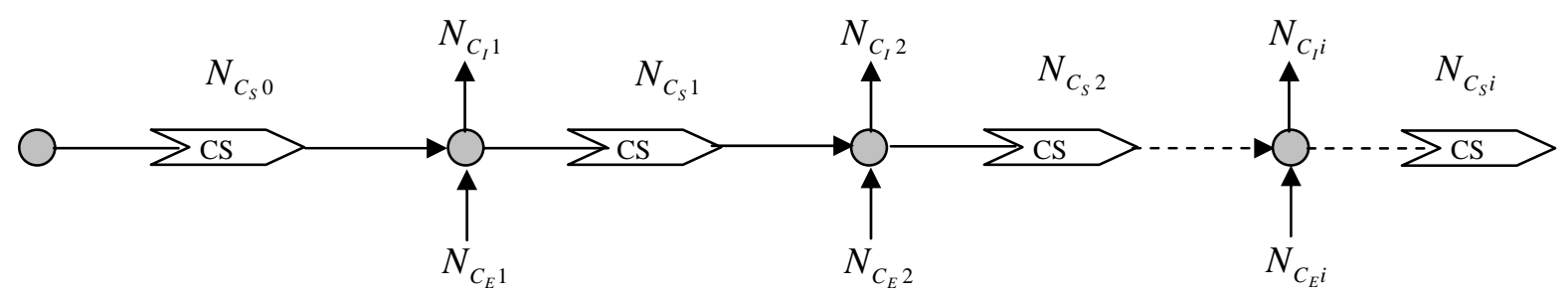

Abbildung 9: Bestimmung des Vollcontaineraufkommens

$$
N_{C R}=\sum_{1}^{k}\left(N_{C_{I} i}+N_{C_{E} i}\right)
$$

pro Hafen gilt:

$$
N_{C_{I} i}+N_{C_{E} i}=\Delta_{i} \cdot \frac{N T E U_{S}}{F_{T E U}} \cdot \alpha
$$

wobei $\Delta_{i}$ die Menge umzuschlagender Container im Hafen i anteilig gemessen an der Schiffsnettoladekapazität ist. Durch $\alpha$ wird wie bereits erwähnt eine evtl. verminderte Auslastung durch geringeres Ladungsaufkommen berücksichtigt. Somit ist die Menge umzuschlagender Container:

$$
N_{C_{R}}=\sum_{1}^{k} \Delta_{i} \cdot \frac{N T E U_{S}}{F_{T E U}} \cdot \alpha=\frac{N T E U_{S}}{F_{T E U}} \cdot \alpha \cdot \sum_{1}^{k} \Delta_{i}
$$


Die Aufsummierung der anteiligen Umschlagmengen pro Hafen und Stellplatz kann auch als Wiederbelegung $W_{R}$ eines Stellplatzes pro Rundreise definiert werden.

$$
\sum_{1}^{k} \Delta_{i}=2 \cdot W_{R}
$$

Die Wiederbelegung 1 pro Stellplatz bedeutet, dass ein Container im Laufe der Rundreise gelöscht und einer geladen wird. Fährt ein Schiff im Rahmen seiner Rundreise z.B. nur zwischen zwei Häfen so kann die Wiederbelegung maximal 2 werden. Vier Umschlagoperationen wären dafür notwendig.

Somit kann die Anzahl aller pro Rundreise umzuschlagender Container $N_{C_{R}}$ und damit notwendigen Umschlagoperationen alleine durch die Schiffsnettoladekapazität in TEU, einem Auslastungsfaktor und einer angenommenen Wiederbelegung eines Stellplatzes bestimmt werden.

$$
N_{C_{R}}=\frac{N T E U_{S}}{F_{T E U}} \cdot \alpha \cdot 2 \cdot W_{R}
$$

Jede Umschlagoperation wird mit einem Kaitarif belegt. Diese sind zum einen für 20' und 40' Container und zum anderen zwischen den Kontinenten verschieden. Die Aufteilung der Umschlagmenge $N_{C_{R}}$ in 20' und 40' erfolgt über:

$$
\begin{aligned}
& N_{C R}=N_{C 20 R}+N_{C 40 R} \\
& N_{C 20 R}=\left(\frac{2 N T E U_{S}}{F_{T E U}}-N T E U_{S}\right) \cdot \alpha \cdot 2 \cdot W_{R} \\
& N_{C 40 R}=\left(N T E U_{S}-\frac{N T E U_{S}}{F_{\text {TEU }}}\right) \cdot \alpha \cdot 2 \cdot W_{R}
\end{aligned}
$$

Bezüglich der Verteilung der Umschlagoperationen zwischen den Kontinenten kann davon ausgegangen werden, dass die Hälfte der Umschlagoperationen einer Rundreise auf einem Kontinent statt findet. Vereinfachend werden pro Kontinent gleiche Kaitarife angenommen. Der Unterschied der Kaitarife lässt sich über einen Faktor $\beta$ ausdrücken, z.B:

$$
K_{K A I_{20} A s i e n}=\beta \cdot K_{K A I_{20} \text { Europa }}
$$

Mit diesen Abstraktionen lassen sich die Zeitkomponenten einer Rundreise mit k-Häfen, ohne sie genau zu kennen, wie folgt berechnen:

$$
\begin{aligned}
& T_{R}=T_{S_{R}}+T_{L R} \\
& T_{R}=\frac{S_{R}}{V_{S}}+k \cdot T_{H}+\frac{N T E U_{s}}{F_{T E U}} \cdot 2 \cdot \alpha \cdot W_{R} \cdot \frac{T_{U_{M}}}{N_{C B}}
\end{aligned}
$$


Darauf aufbauend generieren sich die Kostenkomponenten zu:

$$
\begin{aligned}
& K_{F R}=K_{F S} \cdot\left(\frac{s_{R}}{V_{S}}+n \cdot T_{H}+\frac{N T E U_{s}}{F_{T E U}} \cdot 2 \cdot \alpha \cdot W_{R} \cdot \frac{T_{U_{M}}}{N_{C B}}\right) \\
& K_{V S R}=K_{V S} \cdot \frac{s_{R}}{V_{S}} \\
& K_{V H R}=K_{H S} \cdot k \\
& K_{U R}=\alpha \cdot W_{R} \cdot(1+\beta) \cdot\left(K_{K A I_{20} \text { Europa }} \cdot\left(\frac{2 N T E U_{S}}{F_{T E U}}-N T E U_{S}\right)+K_{K A I_{40} \text { Europa }} \cdot\left(N T E U_{S}-\frac{N T E U_{S}}{F_{T E U}}\right)\right)
\end{aligned}
$$

Sonstige Kosten bleiben in der Betrachtung unverändert.

\subsection{Einnahmenmodell}

\subsubsection{Einnahmenmodell einer Rundreise}

Jeder Containertransport verursacht Kosten. Aber nur volle Container können Einnahmen generieren. Bei der Berechnung der Einnahmen sind jedoch die weltweiten Imbalancen $Q_{R}$ zu berücksichtigen. Imbalancen sind Unpaarigkeiten in den Güterströmen zwischen den Kontinenten. In 2005 wirkten sich diese Unpaarigkeiten auf die Vollcontainerströme wie in Abbildung 10.

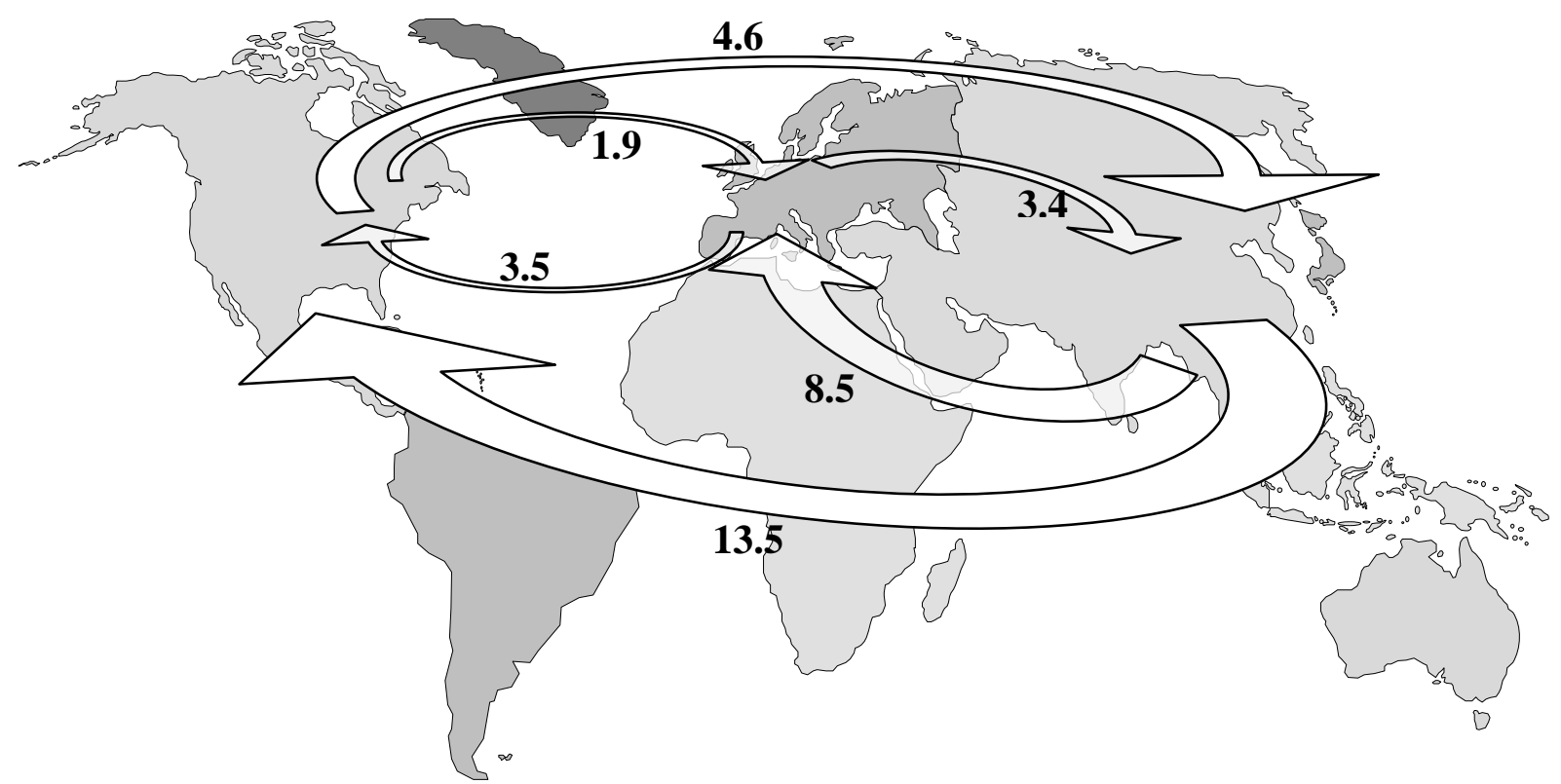

Abbildung 10: Verteilung der Vollcontainerströme in Mio. TEU in 2005 zwischen Europa, Asien und Nordamerika [HAP06, S.21]

Im Transpazifikverkehr lagen die Imbalancen in 2005 immerhin bei 66\%. Durch die Imbalancen ergeben sich nicht nur unterschiedliche Vollcontainerströme sondern auch unterschiedliche Frachtraten je nach Richtung. Entlang der Richtung mit voller Auslastung ist eine deutlich höhere Frachtrate zu erzielen als in die entgegensetzte Richtung.

Imbalancen müssen somit bei der Ermittlung des Vollcontaineraufkommens einer Rundreise $N_{C V R}$ und der zutreffenden Frachtrate berücksichtigt werden. Das Vollcontaineraufkommen einer Rundreise setzt sich dabei aus der Summe der vollen Exportcontainern $N_{C_{E V}}$ pro Hafen i zusammen (Abb. 11). 


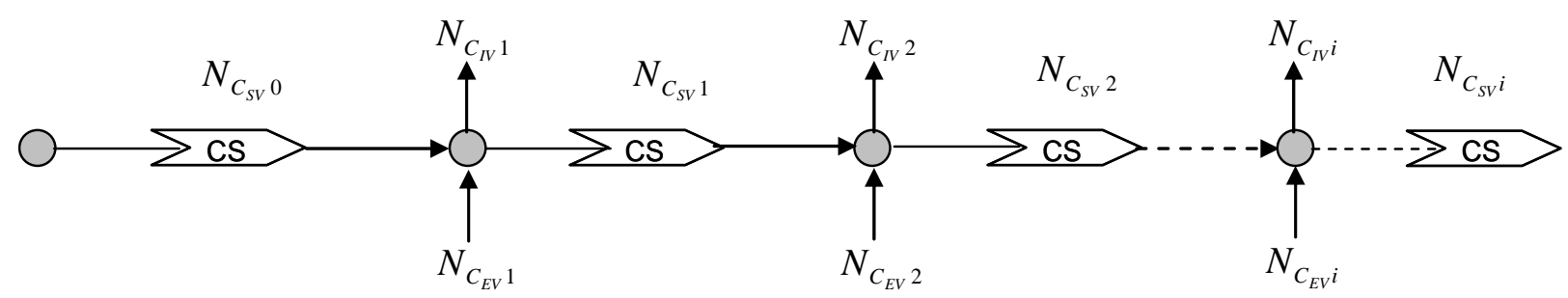

Abbildung 11: Vollcontaineraufkommen einer Rundreise

$$
\begin{aligned}
& N_{C_{S V} i}=N_{C_{S V} i-1}-N_{C_{I V} i}+N_{C_{E V} i} \\
& N_{C_{S V} i} \leq N T E U_{S} \cdot \alpha \cdot\left(100 \%-Q_{R i}\right) \\
& 0 \leq \alpha \leq 1 ; 0 \leq Q_{R i} \leq 100 \% \\
& N_{C V R}=\sum_{1}^{k} N_{C_{E V} i}
\end{aligned}
$$

Bei den Einnahmen sind ebenfalls wieder die Unterschiede zwischen 20’ und 40’ Containern zu berücksichtigen:

$$
\begin{aligned}
& E_{H_{20} i}=N_{C_{E V 20} i} \cdot E_{C_{20}} \\
& E_{H_{40} i}=N_{C_{E V 40} i} \cdot E_{C_{40}} \\
& E_{H i}=E_{H_{20} i}+E_{H_{40} i} \\
& E_{R}=\sum_{1}^{k} E_{H i}
\end{aligned}
$$

Die Einnahmen pro Container $E_{C}$ setzen sich nicht nur aus der reinen Frachtrate zusammen, sondern auch aus dem Bunker-Adjustmentfactor $B A F_{T E U}$, dem Currency-Adjustmentfactor $C A F_{T E U}$ sowie den Terminal Handling Charges THC , die für 20' und 40' Container unterschiedlich ausfallen und für den Versand- und Empfangshafen zu entrichten sind.

Da es auch hier wiederum sehr schwierig ist, die möglichen Einnahmen konkreten Häfen zuzuordnen, wird das Modell ebenfalls dahingehend verändert, dass die Einnahmen ohne eine konkrete Rundreise ermittelt werden können.

\subsubsection{Allgemeines Einnahmenmodell}

Vereinfachend wird auch hier wieder angenommen, dass die Einnahmen pro Container, die auf einem Kontinent erzielbar sind, sich durch ein Verhältnis zu den erzielbaren Einnahmen des anderen Kontinents ausdrücken lassen.

$$
E_{C \text { Asien-Europa }}=\delta \cdot E_{C \text { Europa-Asien }}
$$

Die Menge der Vollcontainer ist gleich der halben Menge der Umschlagoperationen der Rundreise bei Imbalance $0 \%$. Sind Imbalancen vorhanden so reduziert sich im Gebiet der Imbalancen die Vollcontainermenge um die Differenz der Imbalance zur Vollauslastung.

$$
N_{C V R}=\frac{N T E U_{S}}{F_{T E U}} \cdot \frac{\alpha}{2} \cdot W_{R}+\frac{N T E U_{S}}{F_{T E U}} \cdot \frac{\alpha}{2} \cdot W_{R} \cdot\left(100 \%-Q_{R}\right)
$$


Bei der Berechnung der Einnahmen sind wie bereits erwähnt für 20' und 40' Container unterschiedliche Frachtraten $^{3}$ anzusetzen. Im Gebiet der Imbalance reduziert sich jeweils die Frachtrate um den Faktor $\delta$. Die Einnahmen, die sich mit vollen 20' Containern pro Rundreise erzielen lassen, berechnen sich zu:

$$
E_{R 20}=E_{C_{20} \text { Europa-Asien }} \cdot\left(\frac{2 \cdot N T E U_{S}}{F_{T E U}}-N T E U_{s}\right) \cdot \frac{\alpha \cdot W_{R}}{2} \cdot\left(1+\delta \cdot\left(100 \%-Q_{R}\right)\right)
$$

Die Einnahmen der 40' Container sind berechenbar über:

$$
E_{R 40}=E_{C_{40} \text { Europa-Asien }} \cdot\left(N T E U_{S}-\frac{N T E U_{s}}{F_{T E U}}\right) \cdot \frac{\alpha \cdot W_{R}}{2} \cdot\left(1+\delta \cdot\left(100 \%-Q_{R}\right)\right)
$$

Die Summe der beiden Einnahmen pro Containertyp ergeben die Gesamteinnahmen pro Rundreise.

\subsection{Rentabilitäts- und Leistungsbewertung}

\subsubsection{Rentabilitätsbewertung}

Die Rentabilität einer Rundreise ist ein einfaches Maß für die Wirtschaftlichkeit einer Rundreise. Sie ist definiert als Verhältnis von Gewinn zu Kosten.

$$
R_{R}=\frac{E_{R}-K_{R}}{K_{R}}
$$

Für das in Tabelle 1 definierte Schiffsgrößencluster und typischen Parametern einer Asien-Europa Rundreise ergeben sich die in Abbildung 12 dargestellten Rentabilitäten:
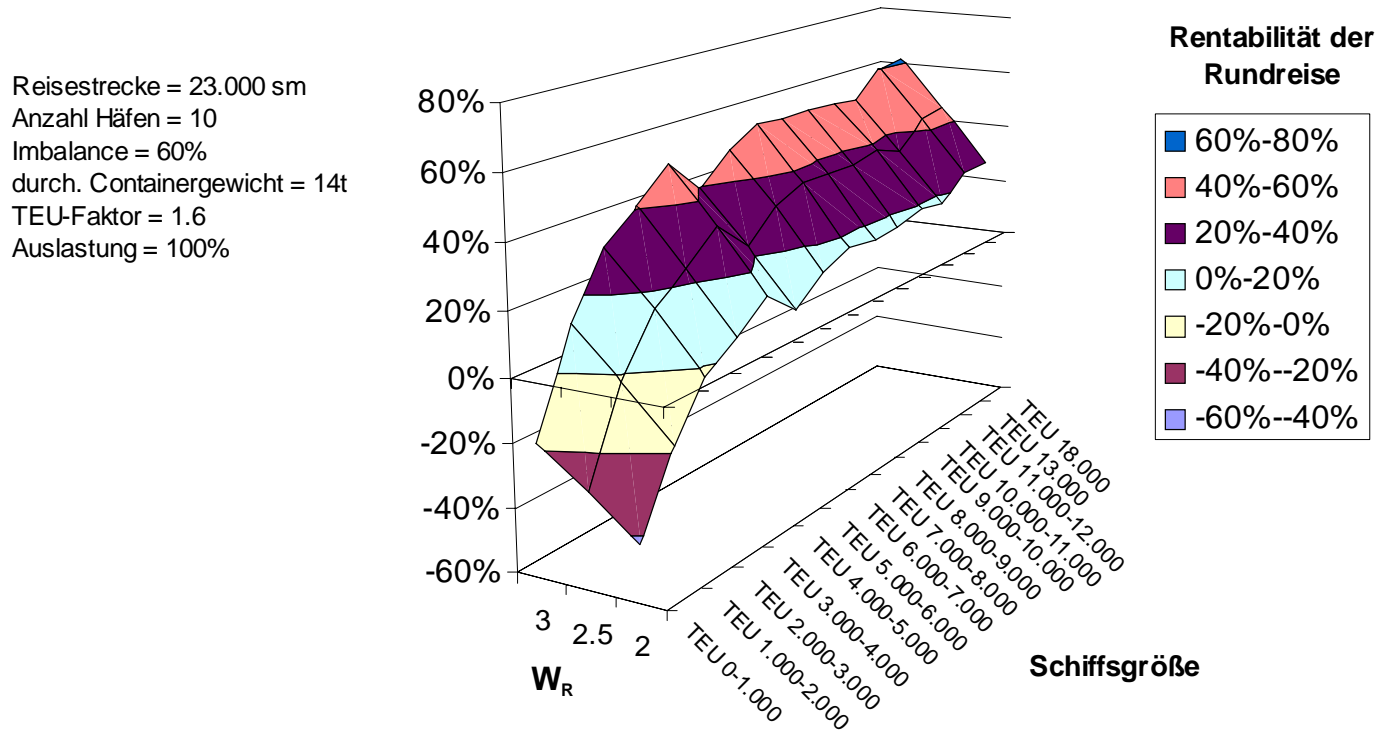

Abbildung 12: Rentabilitätsvergleich zwischen verschiedenen Schiffstypen

Kleinere Schiffe bis ca. 2.000 TEU sind offensichtlich in der heutigen Zeit nicht mehr in der Lage rentabel Asien-Europa-Transporte abzuwickeln. Erst ab ca. 3.000-4.000 TEU stellt sich eine nennenswerte Rentabilität ein. Ab einer Größe von ca. 8.000 TEU stagniert jedoch die Rentabilität und nur im Bereich ab 13.000 TEU steigt sie nochmals leicht an.

\footnotetext{
${ }^{3}$ Frachtrate umfasst hier alle Bestandteile inkl. BAF, CAF und THC 
Gegenwärtig existieren zwei Schiffe mit einer Brutto-Stellplatzzahl von 13.000 TEU. Ob diese Schiffe die in Tabelle 1 angegebene Geschwindigkeit erreichen ist fraglich, da es sich entgegen bisheriger Annahmen für solche Schiffe immer noch um Einschraubenschiffe handelt. Jegliche Verlangsamung führt dazu, dass die Rundreisezeit ansteigt und mehr Fixkosten in der Rentabilitätsbetrachtung zur Geltung kommen. Das 18.000 TEU Schiff ist bisher ein reiner Planungsentwurf [Wij00] und wird deshalb nicht weiter betrachtet.

Der größte Zeitaufschlag für die Rundreisezeit kann aber in den Häfen entstehen. Bei der Betrachtung der Hafenund Seezeiten für eine Rundreise wird deutlich, dass Zeitunterschiede ab ca. 3.000-4.000 TEU fast ausschließlich in den Häfen entstehen (Abb. 13).

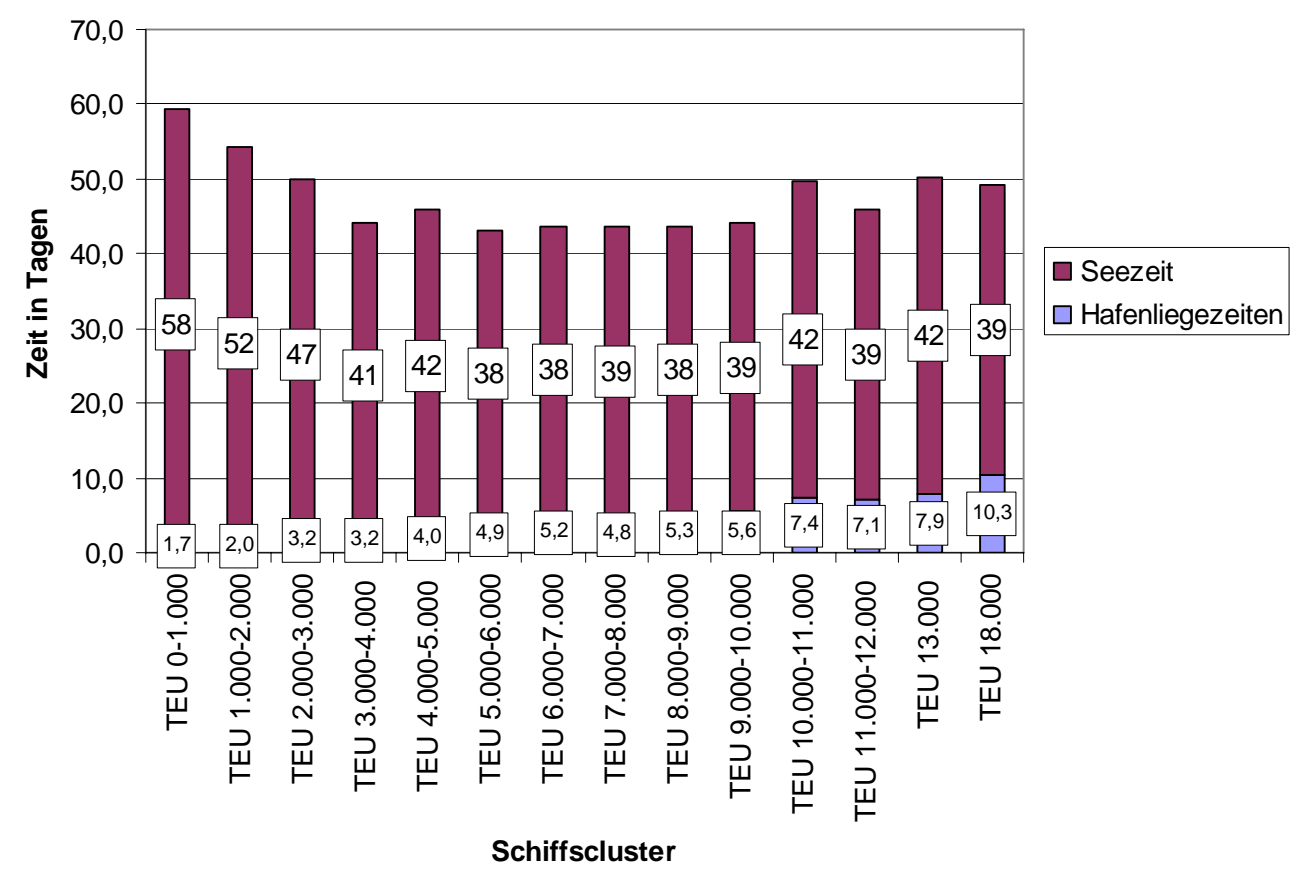

Abbildung 13: Verteilung der See- und Hafenliegezeiten

Bei den Hafenliegezeiten ist anzumerken, dass die hier gewählten Berechnungsverfahren bzw. die einzusetzenden Parameter Spielzeiten und Anzahl Brücken pro Schiffstyp ausschließlich auf Literaturangaben beruhen, die sich mit der Neuplanung von Containerterminals befassen [z.B. Jan87, CUX00]. Reale Messungen im Hamburger Hafen haben ergeben, dass die Liegezeit pro Schiff ca. doppelt so lang ist wie die, die aus den allgemein anerkannten Berechnungsverfahren hervorgehen. Allerdings kann nicht gesagt werden, ob das auch auf andere Häfen zutrifft.

Die Hafenliegezeit ist jedoch ein Parameter, der mit am stärksten die Rentabilität beeinflusst. Kleinere Schiffe, die schneller in den Häfen als größere Schiffe pro umzuschlagenden Container abgefertigt werden, können die größeren Schiffe in der Rentabilität überholen.

Die Betrachtung der Rentabilität an sich, ist aber nicht ausreichend, um die Wirtschaftlichkeit von Containerschiffen zu beurteilen. Die Rentabilitäten wurden wie in Abbildung 13 ersichtlich in unterschiedlichen Zeiträumen erwirtschaftet.

\subsubsection{Leistungsbewertung}

Die Leistung eines Schiffes kann verschieden definiert werden. Im Rahmen dieser Untersuchung wurde als Leistung oder Produktivität die Rentabilität pro Zeiteinheit wie folgt definiert.

$$
P_{R}=\frac{R_{R}}{T_{R}}
$$


Für die in Abbildung 12 angegebenen Rentabilitäten ergeben sich folgende Produktivitäten:

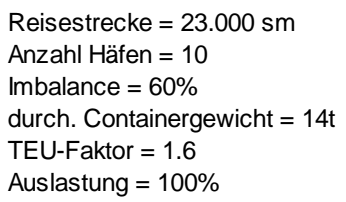

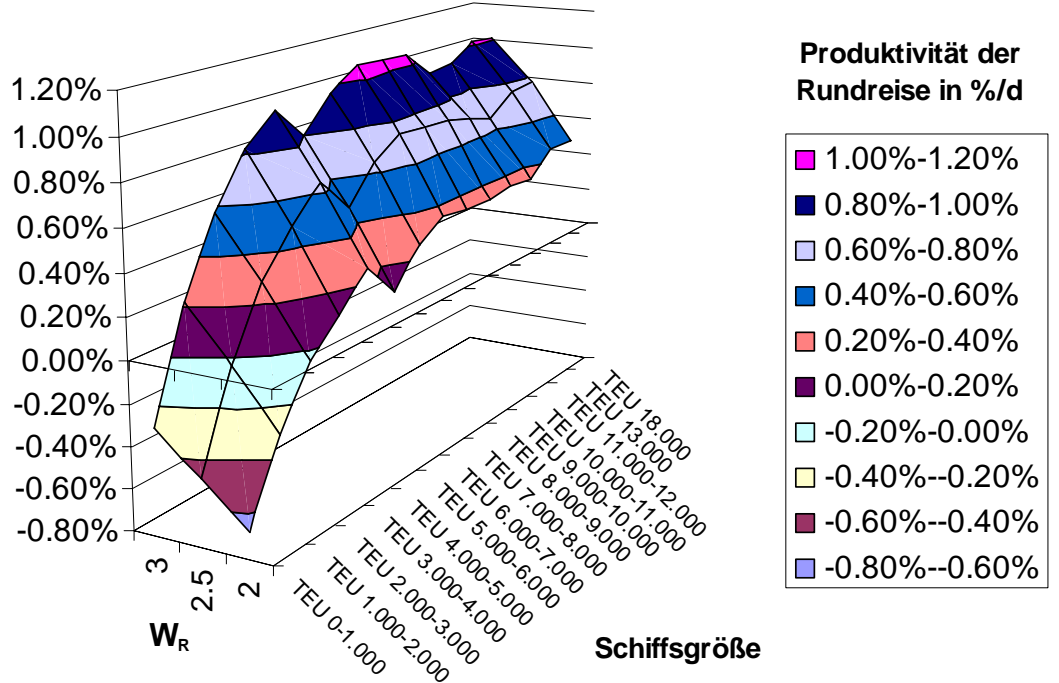

\section{Abbildung 14: Produktivitätsvergleich zwischen verschiedenen Schiffstypen}

Deutlich ist zu erkennen, dass Schiffe im Bereich 7.000-9.000 TEU die höchste Produktivität aufweisen. Pro Zeiteinheit erwirtschaften diese Schiffe relativ gesehen mehr Geld. Die Produktivität kann auch bis auf den Stellplatz eines Schiffes heruntergebrochen werden. Dabei zeigt sich, dass es bei diesen Schiffstypen gelingt den Stellplatz auf häufigsten pro Rundreise zum Einsatz zu bringen. Die Häufigkeit der Nutzung, die Slotproduktivität, ist höher als die größerer oder kleiner Schiffe.

Rentabilität und Produktivität lassen sich durch folgende wesentliche, nichtmonetäre Parameter beeinflussen:

- Containerdurchschnittsgewicht, wirkt auf die Netto-Stellplatzanzahl $N T E U_{S}$

- $\quad$ TEU-Faktor $F_{T E U}$, wirkt auf die Ladungsstruktur und damit Anzahl der Umschlagoperationen

- Imbalance $Q_{R}$, wirkt auf das Vollcontaineraufkommen

- Auslastung $\alpha$, wirkt insgesamt auf das Containeraufkommen

- Wiederbelegung $W_{R}$, wirkt insgesamt auf das Containeraufkommen

- $\quad$ Dauer des Umschlagprozesses $T_{U M}$ pro Brücke, wirkt auf die Hafenliegezeit

- Anzahl einsetzbarer Containerbrücken $N_{C B}$, wirkt auf die Hafenliegezeit

Bis auf den ersten Parameter hat keiner der Parameter irgendetwas mit der Schiffsgröße zu tun. Und selbst der erste Parameter wird durch die Handels- und Ladungsentwicklung bestimmt und liegt nicht im Machtbereich einer Reederei oder Schiffswerft. Insofern ist das Führen der Diskussion über die Wirtschaftlichkeit oder Unwirtschaftlichkeit eines Schiffes allein über die Schiffsgröße überhaupt nicht zielführend, ja sogar thematisch nebensächlich.

Ein bisher hier nicht explizit erwähnter Parameter ist die Anzahl der Häfen einer Rundreise. Für die Umschlaggeschwindigkeit ist die Anzahl der Häfen nicht entscheidend. Es ist egal ob alle Container in einem Hafen oder verteilt über z.B. fünf Häfen geladen und gelöscht werden. Die reine Umschlagzeit ist theoretisch identisch. Lediglich durch Anlaufzeiten und Hafengebühren kann es hier zu leichten Zeitverzögerungen oder Kostenaufschlägen kommen. Diese fallen aber gegenüber der Umschlagzeit und den Kaitarifen deutlich geringer aus.

Allerdings sind bei weitem nicht alle Häfen der Welt in der Lage Großcontainerschiffe abzufertigen. Mit zunehmender Schiffsgröße schränken sich die Anzahl erreichbarer Häfen immer mehr ein. Begrenzende Faktoren sind dabei der Tiefgang der Häfen sowie die erreichbaren Containerreihen durch die Containerbrücken. 
Tabelle 2 gibt einen Überblick über den gegenwärtigen Ausbauzustand führender Häfen in der Welt:

Tabelle 2 Tiefgangsbeschränkungen und Umschlagmöglichkeiten führender Welthäfen nach dem HHM 2006

\begin{tabular}{|c|c|c|c|c|}
\hline Ports & $\begin{array}{l}\text { Weekly } \\
\text { calls }\end{array}$ & $\begin{array}{c}\text { T/S } \\
\text { share }\end{array}$ & $\begin{array}{c}\text { Draft } \\
\text { m. }\end{array}$ & $\begin{array}{c}\text { Gantry } \\
\text { outreach }\end{array}$ \\
\hline Algeciras & 5 & $85 \%$ & 16.0 & 22 \\
\hline Antwerp & 8 & $22 \%$ & 14.0 & 21 \\
\hline Bremerhaven & 6 & $43 \%$ & 14.0 & 22 \\
\hline Busan & 16 & $35 \%$ & 16.0 & 22 \\
\hline Colombo & 15 & $70 \%$ & 14.0 & 18 \\
\hline Damietta & 7 & $80 \%$ & 14.5 & 18 \\
\hline Dubai & 5 & $43 \%$ & 14.0 & 25 \\
\hline Felixstowe & 12 & $20 \%$ & 14.7 & 23 \\
\hline Gioia Tauro & 12 & $95 \%$ & 15.0 & 20 \\
\hline Hamburg & 21 & $46 \%$ & 15.1 & 23 \\
\hline Hong Kong & 57 & $20 \%$ & 15.5 & 23 \\
\hline Jeddah & 15 & $22 \%$ & 16.0 & 20 \\
\hline Kaohsiung & 17 & $55 \%$ & 14.0 & 20 \\
\hline Khor Fakkan & 4 & $70 \%$ & 15.0 & 22 \\
\hline Le Havre & 14 & $30 \%$ & 14.0 & 22 \\
\hline Malta & 8 & $93 \%$ & 15.5 & 19 \\
\hline Nagoya & 5 & n.a. & 14.0 & 18 \\
\hline Ningbo & 14 & n.a. & 17.0 & 23 \\
\hline Piraeus & 5 & $62 \%$ & 14.0 & 18 \\
\hline Port Kelang & 29 & $54 \%$ & 16.5 & 22 \\
\hline Port Said & 11 & $80 \%$ & 16.5 & 22 \\
\hline PTPelepas & 15 & $95 \%$ & 14.0 & 22 \\
\hline Qingdao & 19 & n.a. & 14.5 & 25 \\
\hline Rotterdam & 25 & $24 \%$ & 22.5 & 22 \\
\hline Salalah & 4 & $98 \%$ & 15.0 & 22 \\
\hline Shanghai & 19 & $35 \%$ & 14.2 & 22 \\
\hline Shenzhen & 32 & n.a. & 16.0 & 23 \\
\hline Singapore & 48 & $80 \%$ & 14.8 & 23 \\
\hline Southampton & 9 & $10 \%$ & 14.9 & 20 \\
\hline Taranto & 6 & $95 \%$ & 14.0 & 22 \\
\hline Tokyo & 5 & n.a. & 15.0 & 18 \\
\hline Valencia & 8 & $21 \%$ & 16.0 & 22 \\
\hline
\end{tabular}

Successively shown are the following details:

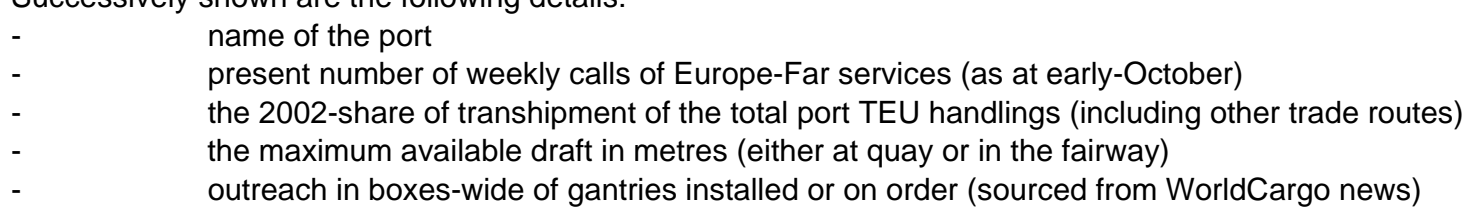

Hinzu kommt noch ein bisher kaum beachteter Faktor, nämlich die Containerlagenhöhe. 9-10 Containerlagen über Deck zu erreichen (13.000 TEU-Schiff bei voller Auslastung), stellt für die Containerbrücken- und Wasserbauer eine gewaltige Herausforderung dar.

Ohne die Ursachen für die Beschränkungen der Häfen näher zu benennen kann im Ergebnis festgehalten werden, dass sich Großcontainerschiffe auf weniger Häfen einschränken müssen als kleinere. Großcontainerschiffe decken damit ein kleineres Fahrtgebiet ab, als kleinere Schiffe. Und das verursacht einen wesentlichen Rückwirkungseffekt auf die Transportkette.

\section{Rückwirkungen der Schiffsgrößenentwicklung auf die Transportkette}

Auf Grund der bisherigen Untersuchungen kann gesagt werden, dass sich der Faktor $\Delta$ aus Abbildung 3 überproportional zur Schiffsgröße entwickeln wird, wenn Großcontainerschiffe eine ähnlich hohe Rentabilität und Produktivität wie kleinere Schiffe aufweisen sollen. Großcontainerschiffe müssen ihre Stellplätze über weniger Häfen genauso oft zum Einsatz bringen wie kleinere Schiffe über mehrere. 
Das geht nur, wenn bei diesen Schiffen pro Hafen mehr Container umgeschlagen werden. Empirische Untersuchungen im Hamburger Hafen stützen diese Aussage (Abb. 15).

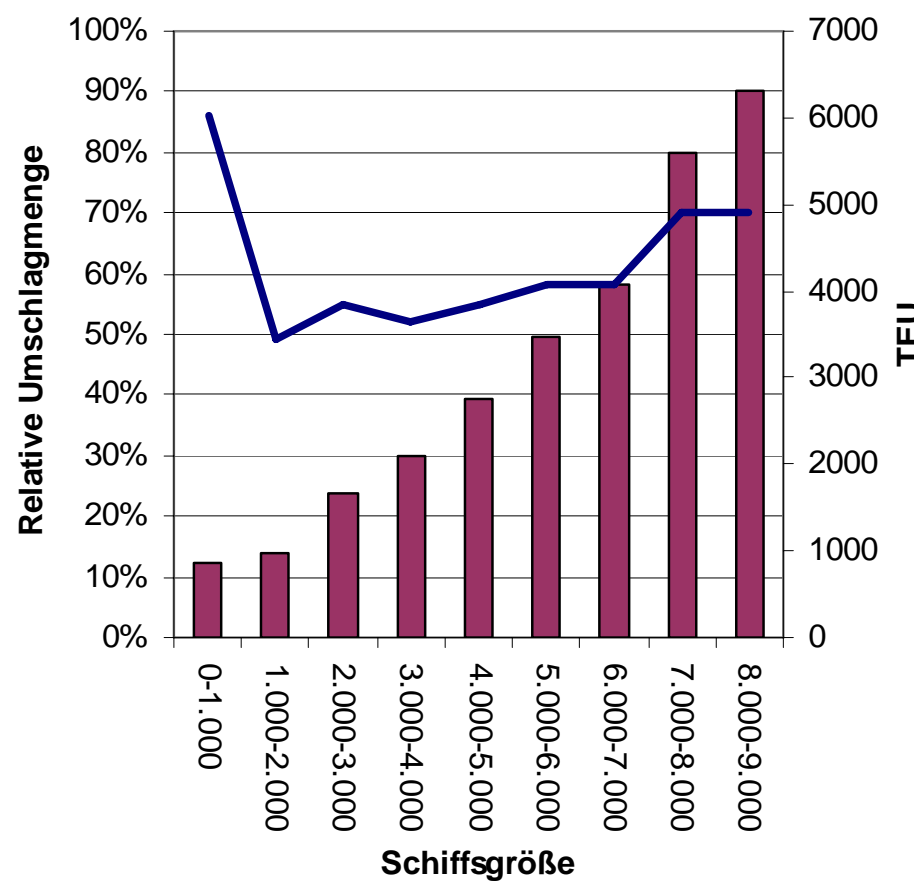

Abbildung 15: Beobachtungen des Faktors $\Delta$ für den Hamburger Hafen (blaue Linie)

Diese Entwicklung wird keine Einzelerscheinung weniger Schiffe bleiben ${ }^{4}$. Wenn sich große Containerschiffe durchsetzen, so werden die Häfen, die mit diesen Schiffen zu tun haben, ein deutlich stärkeres Spitzenaufkommen erwarten. Dieses Spitzenaufkommen an Containern wird neue Anforderung an die Lagerung sowie das Sammeln und Verteilen der Container stellen. Es besteht hier gegenwärtig die Gefahr einer Durchlaufzeitverzögerung der Container, was sich insbesondere bei wertvoller Ladung nachteilig ausdrücken kann.

Aus Grenzwertbetrachtung mit dem Kosten- und Leistungsmodell geht auch hervor, das kombinierte Dienste aus Großcontainerschiffen und kleineren Containerschiffen in Fahrtgebieten, die auch direkt von kleineren Containerschiffen abgedeckt werden können, keine Chance haben. Z.B. Ladung eines Großcontainerschiffes aus Fernost in Rotterdam löschen und dann zum Teil mit einem weiteren Seetransport nach Hamburg zu verschiffen wird zu teuer gegenüber dem Direktanlauf Hamburgs mit einem kleineren Schiff werden. Die geringen Rentabilitätsvorteile der großen Containerschiffe reichen nicht aus, um Folgedienste zu finanzieren.

Im Umkehrschluss heißt das aber, dass Ladung aus Großcontainerschiffen fast ausschließlich für den Hinterlandverkehr sein müssen, wenn die Rentabilität dieser Schiffe größer als die kleinerer sein soll. Die Entwicklung des Spitzenaufkommens an Containern wird sich damit auch im Hinterland fortsetzen.

\footnotetext{
${ }^{4}$ In 2005 wurden ca. 40 Abfahrten von über Schiffen größer als 8.000 TEU im Hamburger Hafen notiert. Insgesamt liefen den Hamburger Hafen in diesem Jahr mehr als 6.000 Containerschiffe an
} 


\section{Literatur}

[Bre93]

[Cul98]

[CUX00]

[Dam06]

[HAP06]

[HWWI06/1]

[HWWI06/2]

[Ihl03]

[Jan87]

[BMBF97]

[Mül05]

[Paw06/1]

[Paw06/2]

[Pawl99]

[Stp02]

[Wij00]

Breitzmann, K.H.; Schönknecht, R: Containerlinienschifffahrt,

Rostocker Beiträge zur Verkehrswissenschaft und Logistik, Eigenverlag der

Universität Rostock, 1993

Cullinane, K.; Khanna, M.: Economies of Scale in Large Container Ships. In: Journal of Transport Economies and Policy 33(1998)02, S.185-208

N.N: CTC Container-Terminal Cuxhaven Machbarkeitsuntersuchung Band I: Erläuterungsbericht Gesamtdarstellung des Vorhabens, Arbeitsgemeinschaft CT Deutsche Bucht Cuxhaven GmbH (i.G.),

Hafenwirtschaftsgemeinschaft Cuxhaven 2000

Damas, P.; Gardiner, P.; Shailesh, G.; Page, M.; Williamson, G.: The Drewry

Container Market Quarterly,

Volume Seven, Third Edition, London, Drewry Shipping Consultant Ltd 2006

N.N: Hapag Lloyd Jahresbericht 2005, www.hapag-lloyd.de, 10.09.2006

A.K: Maritime Wirtschaft und Transportlogistik.

Band A: Perspektiven des maritimen Handels - Frachtschifffahrt und

Hafenwirtschaft, Gemeinsame Studie des HWWI Hamburgische

WeltWirtschaftsinstituts und der Berenberg Bank, Hamburg 2006

A.K: Maritime Wirtschaft und Transportlogistik.

Band B: Perspektiven für maritime Wirtschaft und Transportlogistik -

Strategieansätze aus Unternehmens- und Investorensicht, Gemeinsame Studie des HWWI Hamburgische WeltWirtschaftsinstituts und der Berenberg Bank, Hamburg 2006

Ihlwan, M: Monsters on the high seas.

In: Business Week (2003) Nr,3837-1167, S.37

Jansson, J. O.; Shneerson, D: Liner Shipping Economics.

Champman and Hall, London 1987

Container-Transportsysteme der Zukunft.

Schlussbericht zum gleichnamigen BMBF-Vorhaben, HDW, Kiel 1997

Müller, M.; Schönknecht, A: Kapitalrendite von Großcontainerschiffen, in Internationales Verkehrswesen (2005)9, S.377-

Pawellek, G.; Schönknecht, A: Neue Konzepte zur Beschleunigung intermodaler Transportketten

In: Jahrbuch Logistik 2006, S.14-16

Pawellek, G.; Schönknecht, A: Hinterlandlogistik bringt Kostensenkung im internationalen Hafenwettbewerb.

Schiff \& Hafen (2006)7, S.11-14

Pawlik, T: Seeverkehrswirtschaft, Internationale Containerlinienschifffahrt

Eine betriebswirtschaftliche Einführung.

Gabler, Wiesbaden 1999

Stopford, M: Is the Drive for ever Bigger Containerships Irresistible?

Lloyds List Shipping Forecasting Conference, London 2002

Wijnolst, N.; Waals, F: Malacca-Max [2], Container Shipping Network

Economy. Delft Univ. Press, Delft 2000 